\title{
A Failure Detector for Crash Recovery Systems in Cloud
}

\author{
Bharati Sinha \\ National Institute of Technology, Kurukshetra, India, 136119 \\ E-mail: bharatisinha@nitkkr.ac.in \\ Awadhesh Kumar Singh \\ National Institute of Technology, Kurukshetra, India, 136119 \\ E-mail: aksinreck@ rediffmail.com \\ Poonam Saini \\ Punjab Engineering College, Chandigarh, India, 160012 \\ E-mail: nit.sainipoonam@gmail.com
}

Received: 02 April 2019; Accepted: 19 May 2019; Published: 08 July 2019

\begin{abstract}
Cloud computing has offered remarkable scalability and elasticity to distributed computing paradigm. It provides implicit fault tolerance through virtual machine (VM) migration. However, VM migration needs heavy replication and incurs storage overhead as well as loss of computation. In early cloud infrastructure, these factors were ignorable due to light load conditions; however, nowadays due to exploding task population, they trigger considerable performance degradation in cloud. Therefore, fault detection and recovery is gaining attention in cloud research community. The Failure Detectors (FDs) are modules employed at the nodes to perform fault detection. The paper proposes a failure detector to handle crash recoverable nodes and the system recovery is performed by a designated checkpoint in the event of failure. We use Machine Repairman model to estimate the recovery latency. The simulation experiments have been carried out using CloudSim plus.
\end{abstract}

Index Terms - Failure detectors, Cloud computing, Crash recovery systems, Machine Repairman model.

\section{INTRODUCTION}

Cloud computing has gained massive popularity in recent past owing to its abstraction of resources and on demand services. However, the high availability is essential to support reliable computing. Although, the performance parameters may indicate reliability of hardware components, wide variation in scalability may lead to inaccurate failure estimation. Moreover, the fault tolerance is essential because the computational nodes are highly dynamic. The effectiveness of a fault tolerance mechanism is determined by its accuracy and efficiency in failure detection. Though, cloud is an extension of conventional distributed systems, it has many added features, like high scalability, elasticity, and market driven dynamic pricing, which make it class apart. Hence, the failure detectors (FDs) as in [1] designed for conventional distributed systems are insufficient, inefficient or ineffective in cloud. Therefore, the design of correct Failure Detectors (FDs) for cloud is challenging. The paper presents a prospective implementation of reliable FD in crash-recovery model.

The distributed systems have widely been categorized as synchronous and asynchronous. In general, the synchronous systems use timeout mechanisms to detect failures. However, in a purely asynchronous environment, such timeout mechanism cannot be implemented as there is no bound on any kind of latency. Hence, it is 'impossible' to design and implement failure detector for purely asynchronous environment [2]. The impossibility, here, stems from the fact that it is not possible to distinguish between a crashed node and a very slow node without some time bound on response. Nevertheless, for all practical purposes it is reasonable to consider asynchronous model of computation that allows certain degree of synchrony. The clouds mostly exhibit dynamic asynchronous properties; however, a partial synchronous system can be simulated based on upper bounds of timeconstraint as per QoS requirements.

The FD modules are implemented at each computing node. It detects a crashed node on the basis of time out. Once a node is detected to be crashed it is prohibited permanently from participating in the computation. However, the prohibition is too strong and thus not preferable because a crashed node may recover soon. Thus, another class of FDs, termed unreliable FDs, makes better choice. The unreliable FDs, even after detecting a node suspected, may allow it to participate again provided the node recovers soon. In order to implement this idea, each node maintains a list of correct as well as suspected processes. A node may even fall in suspected list if it has become very slow. Thus, in case of unreliable 
FD, the list of suspected processes maintained at various nodes may not be identical. Conventionally, FDs increment timeout period [3] whenever a process switches from suspect to correct state. However, such a mechanism may instigate an unending movement of nodes from suspect state to correct and vice versa. Generally, such anomalies may be avoided if every FD satisfies the following two properties to a certain degree [1]:

(i) Completeness: FD suspects all crashed processes.

(ii) Accuracy: There is a limit on wrong suspicion by an FD.

Further, above two properties have been reclassified as follows:

> Strong Completeness: All correct processes suspect all crashed processes.

$>$ Weak Completeness: Some correct processes suspect all crashed processes.

> Strong Accuracy: A process is suspected only on crashing.

> Weak Accuracy: Not all correct processes are suspected.

$>$ Eventual Strong Accuracy: After certain time, all correct processes are not suspected by other correct processes

$>$ Eventual Weak Accuracy: After certain time, some correct processes are not suspected by other correct processes.

The above FD mechanism is limited to crash failure only. The system failure estimation is more challenging in crash recovery systems. They need checkpointing at regular intervals to enable rollback recovery. The crash recovery FD performs detection in two steps; firstly, the failed processes are declared crashed as and when they fail. Secondly, after recovery interval, the crashed processes are checked for possible revival. In literature, various methods are prevalent to checkpoint as well as recovery interval estimation. The proposed algorithm can successfully detect if a node fails to respond within timeout interval. In the first step, the crashed node is designated as suspected rather than crashed. Further, if a node fails to respond after the expiry of recovery interval, it is marked as crashed. The checkpoints are maintained to enable the system recovery.

The proposed algorithm considers BCMP [4] mixed multiclass networks for workflow modeling in cloud. The Machine Repairman model [5] is used to estimate checkpointing interval and time to recover. We present an analytical model in order to validate the theoretical accuracy of results. Also, the outcome of simulation experiments is in line with the results of static estimation for cloud environment.

Section 2 presents the relevant literature. Section 3 illustrates the proposed system model. Section 4 explains the analytical model followed by the description of proposed algorithm in section 5. Section 6 presents the proposed algorithm. Section 7 presents experimental validation. In the end, section 8 concludes the work.

\section{RELATED WORK}

The FD in its prevalent form was first proposed by Chandra and Toueg [1]. The authors classified FD into eight categories based upon the type of correctness and accuracy. However, the Chandra-Toueg FD is inherently unreliable as delayed transmission may lead to incorrect detection. Further, based on crash failure assumption, correctness and accuracy have been adapted for recovery systems accordingly. Later, based on the concept of reducibility, Chandra et al. [6] proposed weakest failure detector. Fischer et al. [2] have shown that it is impossible to handle even a single failure in purely asynchronous systems. Nevertheless, the partial synchrony injections make failure detection possible. Dwork et al. [7] have considered two models of synchrony based on (upper and lower) bounds on message transmission delays, speed of processors, and stabilization interval. The message transmission latency and node failures have been observed to follow probabilistic distribution [8]. The registered set of nodes ping each other and if a node does not respond within certain timeout, sender node pings another set of $k$ nodes. These $k$ nodes, in turn, ping the previously nonresponsive node(s). In case of timely response, the initiator node is informed about its presence in the system.

Any service is qualified by Quality of Service (QoS) measures. In literature, several QoS metrics have been proposed to estimate the efficiency of FD [9]. The primary metrics considered are based upon detection latency, time required to correct a faulty detection and frequency of mistaken detection. The proposed metrics are similar to dependency measures. Also, the metrics have been designed considering stochastic behavior using synchronized clocks with no drift. The algorithm considers variable timeout rather than fixed one.

The failure detectors have also been proposed for crash recovery systems by Aguilera et al. [10]. The frequency of failure and revival is maintained to establish the trustworthiness of a node. The suspicion level of a node is enhanced if the failure count continues to increase and it is used to declare failure eventually. However, if the failure count stabilizes, the process is considered trusted one. Secondly, the detection can be performed even without stable storage. The FD for crash recovery system has also been designed using dependability measures for QoS estimation and the node revival has been considered a regenerative process [11].

The early FDs were focused on failure detection mostly for static topology. However, the self-tuning FD (SFD adjusts time out value in view of QoS metrics and other system properties [12]. The implementation proceeds in terms of sliding window where arrival times are used to compute next fresh point interval for each message in sliding window. Turchetti et al. [13] proposed FD for Internet applications. The protocol is capable of managing multiple applications with varying QoS metrics 
simultaneously. It adjusts the timeout interval either by tuning it to maximum requirement or by equating it with GCD of all timeouts for all the components. The assumed bounds are best suited to the remote applications while GCD values are more apt for local applications.

Pannu et al. [14] proposed an adaptive FD for cloud using decision trees. It operates in two phases: adaptive prediction and decision-making. Wang et al. [15] proposed FDKeeper, which has a layer based clustered architecture and takes into account three key parameters namely speed, scalability and heterogeneity. Liu et al. [16] proposed an accrual FD for cloud. Authors use heart beat inter arrival time to compute next timeout interval. However, unlike previous FDs, they advocate Weibull distribution instead of normal or exponential one.

In the literature, many failure detectors have been proposed to detect crash failure. However, crash recovery FD schemes require checkpointing and replication along with efficient detection. Checkpointing has been proposed to be modeled as a service in cloud [17]. VM replication schemes have been used by Mondal et al. [18] for recovery in cloud. Further, reactive recovery algorithms for SDN considering multiple faults have also been proposed [19]. Euclidean based approximation methods have also been used [20]. Markov process based model for failure estimation have also been considered in recovery systems [21]. However most of the previous techniques consider migration based approach for recovery .

The failure in clouds could be at any of the three levels: virtual machine (VM), processing machine (PM) and host Failures at VMs and PMs have been modeled using Continuous Time Markov Chain (CTMC) [22] but the CTMC model suffers from state explosion problem. Nevertheless, our algorithm does not suffer from this problem because we have considered mixed multiclass models for performance modeling.

\section{THE SYSTEM MODEL}

The system consists of two-level hierarchy with broker at the top entrusted with resource management. The computational and system tasks are delegated to datacenters. The server (a.k.a. host) serves as terminal node in the system that performs the computation. The FD module at broker node detects failures in host machines. There are two types of processes, called computational processes and system processes. The computational processes, introduced by broker node, have been modeled as open class of jobs whose arrival and service times follow exponential distribution. The system processes comprise of heartbeat messages that propagate from broker node to the datacenter and further to respective hosts. At any given time, for each host, one heartbeat is under circulation. The successive heartbeat message is initiated only upon successful circulation of the earlier one. Though single heartbeat transmission incurs detection latency, the minimum timeout value compensates for the same. Fig.1 depicts the state diagram of host nodes. Fig.2 illustrates the system architecture.
Further, a host can be in any of the three states: working, failed and recovering.

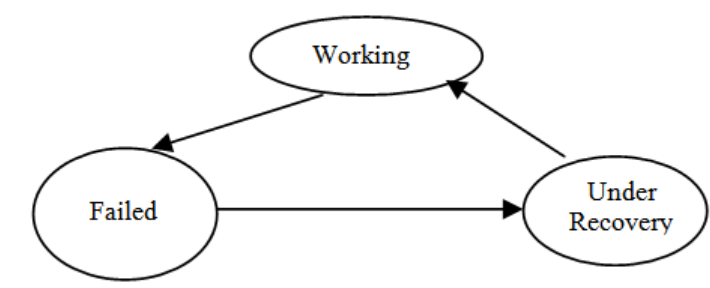

Fig.1. Node States

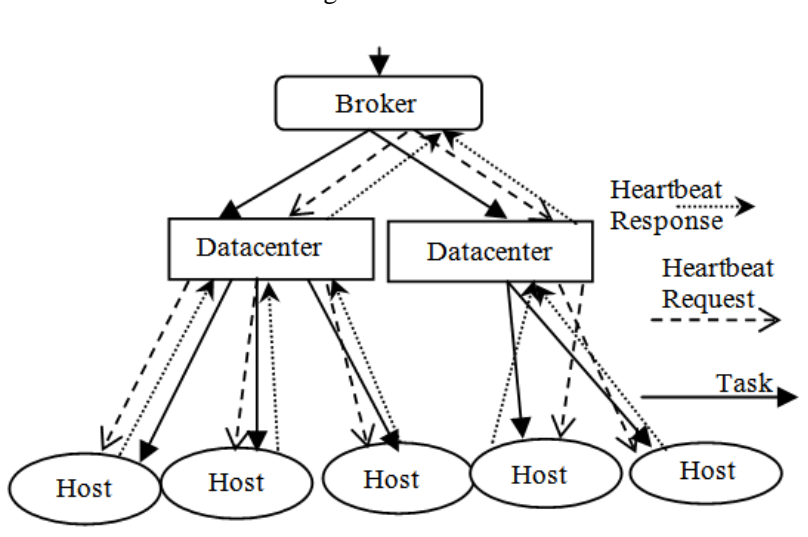

Fig.2. System Architecture

\section{The ANALYTiCAL Model}

The proposed algorithm considers mixed multiclass queuing network [5] to estimate communication latency. The queue size at one level affects the queue size at the next level in a multiplicative manner and thus we have considered product form network. There are two types of tasks, one with an exponential arrival rate and the other continue to circulate in the network in order to establish a mixed network model. The performance parameters are computed by BCMP theorem using Mean Value Analysis (MVA) [23]. The waiting and response time values have been used to estimate task completion time as in [24]. However, our system is crash recovery type, the factors like traffic burst and network congestion, may adversely affect the system performance. The waiting duration at broker includes host recovery time too. Fig. 3 demonstrates host recovery in machine repairman model.

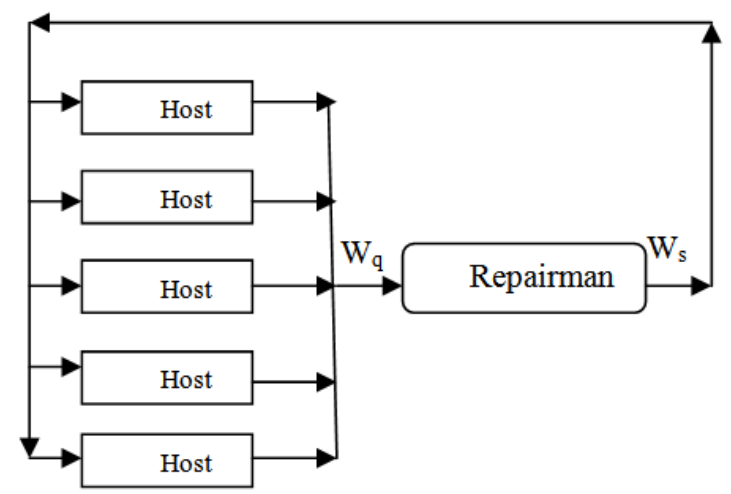

Fig.3. Hosts undergoing recovery 
Table 1 depicts the symbols with their semantics that have been used in our illustration.

Table 1. Symbol Definitions

\begin{tabular}{|c|c|}
\hline Symbols & Semantics \\
\hline$\lambda$ & Average arrival rate of transactions \\
\hline$S_{c k}$ & $\begin{array}{c}\text { Service time per visit of customer of class } c \text { at } \\
\text { node } k\end{array}$ \\
\hline$V_{c \mathrm{k}}$ & $\begin{array}{c}\text { Average no of visits of customer of class } c \text { at } \\
\text { node } k\end{array}$ \\
\hline$D_{c k}$ & Service demand of type $c$ at node $k$ \\
\hline$\rho_{c k}$ & Utilisation of node $k$ by class $c$ \\
\hline$N=\left(N_{1}, N_{2}, \ldots . N_{C}\right)$ & Overall population \\
\hline$W$ & Mean response time \\
\hline$L$ & Average number of customers \\
\hline$C$ & Closed class of customers \\
\hline$O$ & Open class customers \\
\hline
\end{tabular}

The utilisation of server by each customer job is obtained as follows:

i. $\quad$ For every host $k$ :

$$
\rho_{c k}=\lambda_{c} D_{c k}, \text { for all } c \varepsilon\{O\}
$$

ii. $\quad$ Service demand of heartbeat for host $k$

$$
D_{c k}=D_{c k} /\left(1-\rho_{\{o\}, k}\right)
$$

iii. The response time of a heartbeat at host $k$,

$$
W_{c k}[n]=D_{c k}\left[1+L_{k}\left[n-1_{c}\right]\right]
$$

iv. The response time of a particular class $c$ :

$$
W_{c}[n]=\sum_{k=1 t o K} W_{c k}[n]
$$

The above computed response time is valid in a failure free run of the system. However, in the event of failure, recovery time too is taken into account for timeout calculation. Though, the recovery enhances overall system performance, it increases latency. Thus, a task operating on a recovery node would require larger duration for completion as compared to a task allocated to non-recovery node. Therefore, job completion time involves failure duration, recovery interval, and the time required to execute all queued jobs. The recovery time is computed using machine repairman model. The repair time at Repairman is the time required for host recovery. Table 2 describes the semantics of symbols used in the machine repairman model.
Table 2. Symbol Definitions

\begin{tabular}{|c|c|}
\hline Symbols & Meaning \\
\hline$M$ & Mean time to failure \\
$K$ & Number of hosts \\
$W_{r}$ & Time spent in repair \\
$W_{q}$ & Time spent in queue for repair \\
$W_{s}$ & Time spent in service \\
\hline
\end{tabular}

The repairman utilisation factor $(\rho)$ and arrival rate $(\lambda)$ is given by:

$$
\begin{gathered}
\rho=1-p_{0}=B[K, z] \\
\lambda=\rho / W_{s}
\end{gathered}
$$

where, $\mathrm{B}[\mathrm{K}, \mathrm{z}]$ is Erlang's loss formula , $\mathrm{z}=M / W_{s}$

The arrival rate at repairman is used to compute cumulative waiting time at repairman, which includes mean time to failure, waiting time before repair, and service time to repair. It can be computed as follows:

Average arrival rate:

$$
\lambda=K /\left(M+W_{q}+W_{s}\right)
$$

Since,

$$
W_{r}=W_{q}+W s
$$

Thus,

$$
W_{r}=(K / \lambda)-M
$$

The repairman waiting time value obtained from eq (8) is recovery interval considered in case of failure and subsequent recovery. Therefore, the new timeout in case of recovery would be computed as follows:

$$
T_{\text {new }}=2 W_{c}+W_{r}
$$

where, $W_{c}$ is the value obtained from eq (4) for closed class of jobs (i.e. heartbeat).

\section{THE AlgORITHM CONCEPT}

A recoverable node could be in any of the three states: working, failed and recovering. After every timeout interval, non-responding node is added to suspected list. The timeout here is twice of response time for heartbeats considering maximum possible message propagation 
latency. A node once detected as failed is inserted in the suspected list till expiry of recovery interval. Each node in suspected list is checked for possible recovery. The recovered nodes are added in the list of working nodes and heartbeats are resumed. Thus, the total detection latency in crash recovery system is sum of timeout interval and recovery interval. Furthermore, once a node is suspected, no task is allocated to it until its revival. This improvises accuracy in being able to tolerate larger delay. Nevertheless, the node is detected crashed, in case, there is any further delay in the execution. Meanwhile, the system snapshots are taken at the interval same as the mean time between failures so that on occurrence of crash, execution resumes from the state just before the crash point. The broker reschedules the tasks that were scheduled initially on a node that was suspected later. Table 3 describes the events and functions used in proposed algorithm.

Table 3. Events/Functions used in algorithm

\begin{tabular}{|c|c|}
\hline Function/Events & Description \\
\hline initialise_host (h); & $\begin{array}{c}\text { Create hosts with provided } \\
\text { specifications. }\end{array}$ \\
\hline add(h->host_list); & $\begin{array}{l}\text { Create a list of hosts available with } \\
\text { each datacenter. }\end{array}$ \\
\hline mount_vm(); & Mount VMs on hosts available. \\
\hline job_creation; & $\begin{array}{l}\text { Create tasks according to arrival rate } \\
\text { of tasks. }\end{array}$ \\
\hline initialise_datacenter & $\begin{array}{l}\text { Event sent from broker to datacenter } \\
\text { to initiate execution }\end{array}$ \\
\hline assign_task(c) & $\begin{array}{l}\text { Assign task to VMs for execution } \\
\text { according to space shared policy. }\end{array}$ \\
\hline add(c->task_list) & $\begin{array}{l}\text { Create a list of tasks to be executed by } \\
\text { the system. }\end{array}$ \\
\hline allocate_heartbeat $(h)$ & $\begin{array}{l}\text { Schedule one heartbeat at each of the } \\
\text { tasks. }\end{array}$ \\
\hline check_progress() & $\begin{array}{l}\text { Checks the progress of the heartbeat at } \\
\text { each of the host. }\end{array}$ \\
\hline update_pulse & $\begin{array}{l}\text { Sends an event to broker to update } \\
\text { about status of each heartbeat. }\end{array}$ \\
\hline update_pulse & $\begin{array}{l}\text { Sends an event to broker to update } \\
\text { about status of suspected node. }\end{array}$ \\
\hline check_pulse() & $\begin{array}{c}\text { Sends an event from broker to } \\
\text { datacenter to query about the status of } \\
\text { heartbeat progress. }\end{array}$ \\
\hline check_crash() & $\begin{array}{l}\text { Sends an event from broker to } \\
\text { datacenter to query about the status of } \\
\text { heartbeat progress in a suspected } \\
\text { node. }\end{array}$ \\
\hline check_task_status() & $\begin{array}{c}\text { Returns the status of the tasks } \\
\text { allocated. }\end{array}$ \\
\hline task_replication() & $\begin{array}{l}\text { Event sent from broker to datacenter } \\
\text { upon timeout to take snapshot of task } \\
\text { state still running on system. }\end{array}$ \\
\hline replicate_task(); & $\begin{array}{l}\text { Pending tasks after a given timeout } \\
\text { are replicated and scheduled by the } \\
\text { broker. }\end{array}$ \\
\hline add(h->suspect_list); & $\begin{array}{l}\text { The detected nodes are enlisted in the } \\
\text { suspected category upon timeout. }\end{array}$ \\
\hline suspect_count(h) & $\begin{array}{l}\text { List to maintain count of suspected } \\
\text { nodes. }\end{array}$ \\
\hline add(h->crashed_list); & $\begin{array}{l}\text { List of crashed nodes is updated after } \\
\text { recovery interval. }\end{array}$ \\
\hline crashed_count(h) & $\begin{array}{c}\text { List to maintain count of crashed } \\
\text { nodes. }\end{array}$ \\
\hline stop_execution(); & $\begin{array}{c}\text { Detection is stopped when either all } \\
\text { tasks are completed or all hosts have } \\
\text { failed. }\end{array}$ \\
\hline
\end{tabular}

\section{AlgORITHM}

\section{A. Algorithm for broker node}

The proposed algorithm for failure detection considering possible recovery at broker node is as follows:

Initiate computation:

initialise_datacenter();

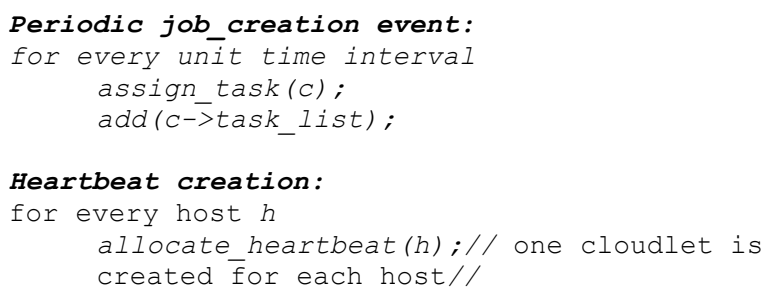

Periodic detection event:

After every timeout interval delay

for every host $h$

check pulse();

task_replication();

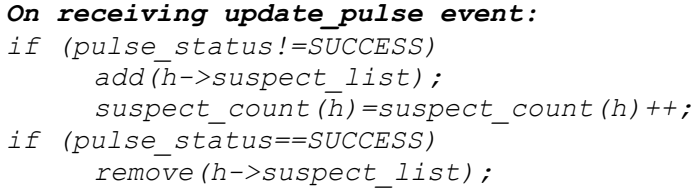

\section{B. Algorithm for datacenter node}

Algorithm for failure detection considering possible recovery at datacenter node is as follows:

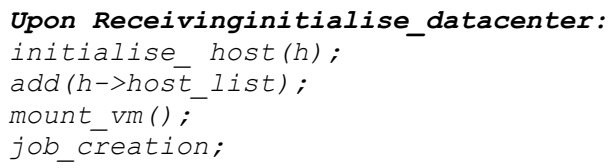

After every periodic interval :

inject fault();//(considering mean failure distribution)

On receiving check pulse event:

check_progress ();

update_pulse;

On receiving check_crash event:

check progress (),

update_crash; 
On receiving task replication event:

for every task $C$

check task status();

if (task stâtus!=SUCCESS)

replicate_task();

\section{EXPERIMENTAL SETUP}

The above mentioned analytical model was computed for the following set of values provided in Table 4 .

Table 4. Simulation values

\begin{tabular}{|c|c|c|c|}
\hline Hosts & 10 & 50 & 100 \\
\hline$\lambda$ & 10 & 50 & 100 \\
\hline $\mathrm{N}_{\mathrm{C}}$ & 10 & 50 & 100 \\
\hline $\mathrm{D}_{\mathrm{o}}$ & 0.05 & 0.01 & 0.005 \\
\hline $\mathrm{D}_{\mathrm{c}}$ & 0.5 & 0.1 & 0.05 \\
\hline \multicolumn{4}{|c|}{ Parameters required for Recovery } \\
\hline $\mathrm{M}$ & $72 \mathrm{~s}$ & $360 \mathrm{~s}$ & $720 \mathrm{~s}$ \\
\hline $\mathrm{W}_{\mathrm{s}}$ & $10 \mathrm{~s}$ & $10 \mathrm{~s}$ & $10 \mathrm{~s}$ \\
\hline
\end{tabular}

Using above values, the following performance parameters are computed:

i. $\quad$ Response time for heartbeat using eq (4).

ii. In case of a crash followed by recovery, total time spent in recovery is computed using eq (8).

iii. From above calculated parameters, timeout value in case of a crash followed by recovery is computed from eq.(10).

The proposed model is implemented using CloudSim plus [25], which is an event driven tool and closely resembles distributed computing environment. Table 5 briefly describes basic terminologies used in our simulation:

Table 5. Basic Terms

\begin{tabular}{|c|c|}
\hline Terms & Functionality \\
\hline Broker & $\begin{array}{c}\text { Resource management entity. Initiates tasks into } \\
\text { the system. }\end{array}$ \\
\hline Datacenter & Entity responsible for working of physical hosts. \\
\hline Host & Execution node where tasks are performed. \\
\hline VM & Virtualization level to emulate resource scaling. \\
\hline Cloudlet & Task to be executed by the system. \\
\hline
\end{tabular}

Following fig. 4 depicts the actual time of crash along with the detected time for 10 hosts.

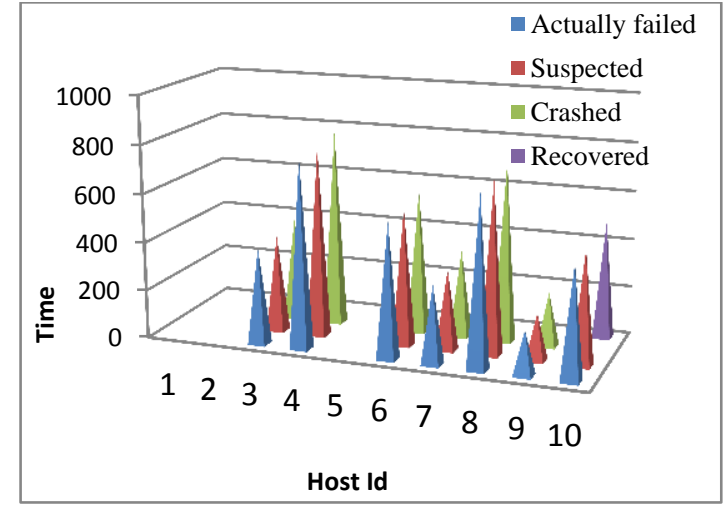

Fig.4. Detection time for each host

Blue cones in fig. 4 represents time of occurrence of failure, red indicates time when they are suspected, green cones are for time of crash declaration whereas violet ones are to indicate recovery. As is evident from fig. 4 there are no cases of false detection or missed detection, since a blue cone precedes the red and green ones in all the cases. Thus, ensuring that a failure is detected, in case, it occurs. Further, the proposed algorithm has been simulated when scaled to 50 and then 100 hosts. It has been observed that completeness and accuracy is preserved even after scaling of resources. Fig. 5 and fig. 6 illustrates the simulation results for 50 and 100 hosts respectively. The occurrence of blue cones before red and red cones before green in fig.5. and fig.6. indicates the sequence of detection mechanism. Hence, a node is suspected when failure occurs and is declared crashed only if it fails to respond within timeout interval. Thus, our algorithm satisfies preliminary requirements of accuracy and completeness that is essential for any failure detection mechanism.

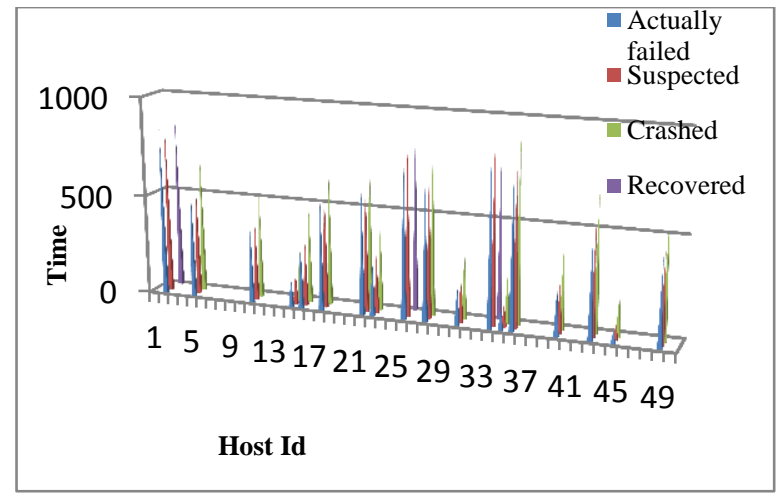

Fig.5. Detection time for 50 hosts 


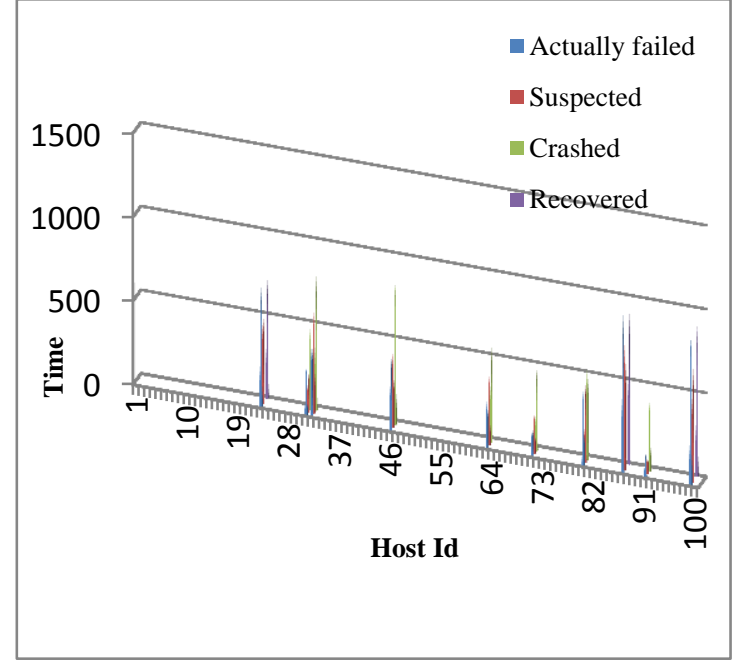

Fig.6. Detection time for 100 hosts

\section{CONCLUSION}

The proposed algorithm considers failure detection problem in crash recoverable cloud nodes. We have abstracted the recovery part though it uses an underlying checkpointing mechanism. In simulation, we assumed jobs having common service requirement however it can also handle jobs with diverse service needs because we have considered mixed multiclass BCMP networks during experimentation. Secondly, in BCMP networks, the response time does not merely reflect detection latency rather it also includes recovery interval. Therefore, our time out calculation is more realistic as well as methodical and more importantly its value is minimal. The algorithm satisfies safety as well as liveness requirements. Our simulation results confirm that there is no false detection. Furthermore, the latency beyond timeout guarantees truthful fault detection. However, the impact of inexplicable traffic burst on the precise fault detection needs further investigation.

\section{REFERENCES}

[1] T.D.Chandra and S. Toueg, "Unreliable failure detectors for reliable distributed systems," Journal of the ACM (JACM), vol.43(2), pp. 225-267,1996.

[2] M. J. Fischer, N. A. Lynch and M. S. Paterson, "Impossibility of distributed consensus with one faulty process," Journal of the ACM (JACM), vol. 32(2), pp. 374-382, 1985.

[3] M. Larrea, A. Fernández and S. Arévalo, "Eventually consistent failure detectors," In Proceedings 10th Euromicro Workshop on Parallel, Distributed and Network-based Processing. IEEE, 2002.

[4] F. Baskett, K. Chandy, R. Muntz, and F. Palacios, "Open, Closed,and Mixed Networks of Queues with Different Classes of Customers," Journal of the ACM, vol. 22(2), pp.248-260, 1975.

[5] A. O. Allen, "Probability, Statistics, and Queuing Theory with Computer Science Applications," second edition, Academic Press, Inc., Boston, Massachusetts, 1990.

[6] T. D. Chandra, V. Hadzilacos and S. Toueg, "The weakest failure detector for solving consensus," Journal of the
ACM (JACM), vol.43(4), pp. 685-722, 1996

[7] C. Dwork, N. Lynch and L. Stockmeyer, "Consensus in the presence of partial synchrony," Journal of the ACM (JACM), vol.35(2), pp.288-323, 1988.

[8] I. Gupta, T. D. Chandra and G. S. Goldszmidt, "On scalable and efficient distributed failure detectors," In Proceedings of the twentieth annual ACM symposium on Principles of distributed computing, pp. 170-179, 2001.

[9] W. Chen, S. Toueg and M. K. Aguilera, "On the quality of service of failure detectors," IEEE Transactions on computers, 51(1), 13-32, 2002.

[10] M. K. Aguilera, S. Toueg and B. Deianov, "Revisiting the weakest failure detector for uniform reliable broadcast," In Proceedings of the 13th International Symposium on Distributed Computing, pp.19-33,1999.

[11] T. Ma, J. Hillston and S, Anderson, "On the quality of service of crash-recovery failure detectors", IEEE Transactions on Dependable and Secure Computing, vol.7(3), pp.271-283, 2010.

[12] N. Xiong, A.V. Vasilakos, J. Wu, Y.R. Yang, A. Rindos, Y. Zhou, W. Song and Y. Pan, "A self-tuning failure detection scheme for cloud computing service," In Parallel \& Distributed Processing Symposium (IPDPS), pp. 668-679, 2012.

[13] R. C. Turchetti, E. P. Duarte, L. Arantes, and P. Sens, “A QoS-configurable failure detection service for internet applications," Journal of Internet Services and Applications, vol.7(1), 2016.

[14] H. S. Pannu, J. Liu, Q. Guan and S. Fu, "AFD: adaptive failure detection system for cloud computing infrastructures," Performance Computing and Communications Conference (IPCCC), IEEE, pp. 71 - 80, 2012.

[15] F. Wang, H. Jin, D. Zou, and W. Qiang, "A Quick and Open Failure Detector for Cloud Computing System," In Proceedings of the International Conference on Computer Science \& Software Engineering, ACM, 2014.

[16] J. Liu, Z. Wu, J. Wu, J. Dong, Y. Zhao and D. Wen, “A Weibull distribution accrual failure detector for cloud computing," PloS one, vol.12(3), 2017.

[17] Cao, Jiajun, et al. "Checkpointing as a service in heterogeneous cloud environments,"2015 15th IEEE/ACM International Symposium on Cluster, Cloud and Grid Computing. IEEE, 2015.

[18] S. K. Mondal, F. Machida, J. K. Muppala, “ Service Reliability Enhancement in Cloud by Checkpointing and Replication," In Principles of Performance and Reliability Modeling and Evaluation, Springer, pp. 425448, 2016.

[19] H. Amarasinghe, A. Jarray, and A. Karmouch, "Faulttolerant IaaS management for networked cloud infrastructure with SDN," IEEE International Conference on Communications (ICC). IEEE, 2017.

[20] C. Yang, X. Xu, K. Ramamohanrao and J. Chen, "A Scalable Multi-Data Sources based Recursive Approximation Approach for Fast Error Recovery in Big Sensing Data on Cloud," IEEE Transactions on Knowledge and Data Engineering, 2019.

[21] L. Luo, S. Meng, X. Qiu and Y. Dai, "Improving Failure Tolerance in Large-Scale Cloud Computing Systems," IEEE Transactions on Reliability, 2019.

[22] Al-Sayed, Mustafa M., Sherif Khattab, and Fatma A. Omara, "Prediction mechanisms for monitoring state of cloud resources using Markov chain model," Journal of Parallel and Distributed Computing, vol.96, pp. 163$171,2016$.

[23] E. Lazowska, J. Zahorjan, G. Graham and K. Sevcik, 
"Quantitative System Performance Computer System Analysis UsingQueueing Network Models," Prentice-Hall, 1984.

[24] B. Sinha, A. K. Singh and P. Saini, "Failure detectors for crash faults in cloud," Journal of Ambient Intelligence and Humanized Computing, 2018.

[25] M. C. Silva Filho, R. L. Oliveira, C. C. Monteiro, Pedro. R. M. Inacio, Manoel, "CloudSim plus: a cloud computing simulation framework pursuing software engineering principles for improved modularity, extensibility and correctness," FIP/IEEE Symposium on Integrated Network and Service Management (IM), IEEE, 2017.

\section{Authors' Profiles}

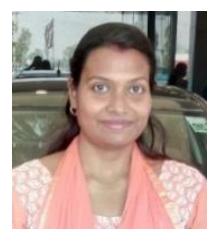

Bharati Sinha received her Bachelor of Technology (B.Tech.) degree in Computer Science Engineering from Bhagalpur College of Engineering, Bhagalpur, Bihar, India in 2010 and her M.Tech. from NIT Rourkela, India in 2012. She joined as Assistant Professor at the Department of Computer Engineering (COE) at the National Institute of Technology, Kurukshetra, India, in 2013 and is pursuing part-time Ph.D. from the Department of Computer Engineering at National Institute of Technology, Kurukshetra, India. Her research interests include cloud computing and distributed systems.

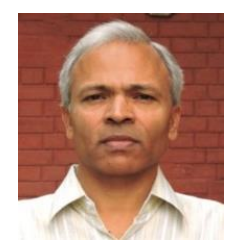

Awadhesh Kumar Singh received his Bachelor of Technology (B.Tech.) degree in Computer Science from Madan Mohan Malaviya University of Technology, Gorakhpur, India, in 1988, and his MTech and Ph.D. degrees in Computer Science from Jadavpur University, Kolkata, India, in 1998 and 2004, respectively. He joined the Department of Computer Engineering at the National Institute of Technology, Kurukshetra, India, in 1991, where he is presently a Professor. Earlier, he also served as head of the Computer Engineering Department during 2007-2009 and 2013-2015. His research interests include distributed algorithms, mobile computing and radio networks.

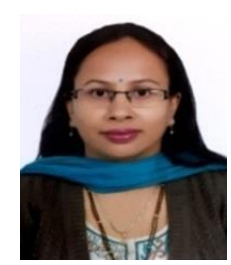

Poonam Saini received her Ph.D. degree in Computer Engineering from National Institute of Technology, Kurukshetra, India in 2013 and M.Tech from UIET, Kurukshetra University, Kurukshetra, India in 2006. She has received B. Tech. in Information Technology from Kurukshetra University, Kurukshetra, India in 2003.

She is currently working as Assistant Professor in Computer Science and Engineering at PEC University of Technology (formerly Punjab Engineering College), Chandigarh, India. Her research interest includes Fault-Tolerant Distributed Computing Systems, Mobile Computing, Ad hoc Networks, Wireless Sensors Networks, Cloud Computing and Security.

How to cite this paper: Bharati Sinha, Awadhesh Kumar Singh, Poonam Saini, "A Failure Detector for Crash Recovery Systems in Cloud", International Journal of Information Technology and Computer Science(IJITCS), Vol.11, No.7, pp.9-16, 2019. DOI: 10.5815/ijitcs.2019.07.02 